Voix et Images

voixetimages

\title{
Entretien avec Claire Martin
}

\section{André Ricard}

Volume 29, numéro 1 (85), automne 2003

\section{Claire Martin}

URI : https://id.erudit.org/iderudit/007536ar

DOI : https://doi.org/10.7202/007536ar

Aller au sommaire du numéro

\section{Éditeur(s)}

Université du Québec à Montréal

\section{ISSN}

0318-9201 (imprimé)

1705-933X (numérique)

Découvrir la revue

Citer cet article

Ricard, A. (2003). Entretien avec Claire Martin. Voix et Images, 29(1), 15-31.

https://doi.org/10.7202/007536ar d'utilisation que vous pouvez consulter en ligne.

https://apropos.erudit.org/fr/usagers/politique-dutilisation/ 


\author{
E N T R E T I E N \\ avec Claire Martin \\ $+++$
}

ANDRÉ RICARD

Secrétaire général de l'Académie des lettres du Québec et écrivain

VoIX ET IMAGES Claire Martin, si vous jetez un regard en arrière, estimez-vous avoir à peu près suivi le parcours que vous vous traciez à vos débuts?

CLAIRE MARTIN D'abord, je ne pensais jamais vivre aussi vieille. Quand j'étais jeune, 85 ans ${ }^{1}$ ça me semblait synonyme de cachexie, de débilité... Mais je me rends bien compte qu'on peut survivre à des idées aussi horribles. Oui, la vie que j'ai menée, c'est à peu près la vie que j'imaginais, que je comptais vivre quand les choses se sont stabilisées, que je suis entrée vraiment dans ma vie, ma vie de travail, ma vie de mariage. J'ai eu une grosse déception, pourtant. Mon mari avait une grand-mère qui est morte à 103 ans. Et je me disais: "Ah! Il y a de la longévité par là; il va être là pour longtemps.» Et puis non, il est mort à 78 ans. J'ai pris ça comme une trahison. Autrement... je vis mon veuvage de façon calme, quand même, paisible. Je suis une femme heureuse. Si on a une disposition au bonheur, je pense qu'on la traîne avec soi toute sa vie.

Voix ET IMAGES Vous êtes sans doute trop douée pour le bonheur. C'est à quoi vous dites qu'il faut attribuer ce long silence dans votre production: vous étiez trop occupée à être heureuse. Il fallait alors que ce soit une disposition de votre tempérament? Parce que votre enfance ne vous aurait pas communiqué cette aptitude au bonheur...

CLAIRE MARTIN Je ne crois pas que mon enfance aurait pu changer quoi que ce soit. Au contraire! C'est quand on est privé de bonheur pendant des années et puis tout à coup qu'il est à portée de la main, qu'on l'apprécie. Et d'autant plus. On sait alors l'apprécier plus que n'importe qui.

VoIX ET IMAGES Après un silence prolongé est paru un recueil de nouvelles que vous avez intitulé Toute la vie. Votre plume, qui est restée longtemps inemployée, a-t-elle grincé au moment de recommencer l'écriture de fiction?

CLAIRE MARTIN Cela s'est fait en douceur. Le premier texte que j'ai écrit après mon retour au Canada, c'est pour Gilles Pellerin, pour le livre sur les écrivains et la ville de Québec. Puis Jean-Guy Pilon s'est mis à me poursuivre de ses mises en demeure: "Il me faut un texte.» Ensuite Louise Maheux-Forcier, qui s'occupe des Écrits: elle en voulait d'autres. À force d'avoir des gens qui me poussaient, qui me téléphonaient, je me suis laissé faire douce violence, jusqu'au moment où Gilles Dorion (il est très autoritaire, vous savez) m'a 
dit: «Je veux rassembler tout ce que vous avez publié dans les revues au cours des années pour faire éditer un volume de nouvelles.» Il l'a fait. Puis il m'a demandé: "Il me faudrait un inédit ou deux, puis deux ou trois, puis trois ou quatre.» On a su compter jusqu'à cinq.

Voix ET IMAGES Lorsque Gilles Dorion a rassemblé les nouvelles éparses de Toute la vie et qu'il vous les a données à lire, vous semblaient-elles conformes à vos exigences d'à présent? Avez-vous été tentée de les remettre en chantier?

CLAIRE MARTIN Non, je suis à l'abri de ce genre de choses. Parce que je suis d'un naturel paresseux. C'est fait, on n'y touche plus. Parfois je me disais: j'aurais pu dire ça autrement, j'aurais pu faire plus bref. De temps en temps, je trouvais que ça faisait un peu gnan-gnan. Mais j'ai toujours été portée à trouver ce que j'écris gnan-gnan.

Voix ex IMAGES Toute la vie est constitué de nouvelles assez éloignées en dates et de pièces récentes. Estimez-vous, en considérant l'ensemble, que votre manière a changé? CLAIRE MARTIN Elle a changé jusqu'à un certain point. Mais il y a plusieurs lecteurs qui m'ont dit: «Non, vous n'avez pas changé. On vous reconnaît tout de suite.» Moi, je pense que j'écris beaucoup plus bref. Qu'il y a beaucoup plus de sous-entendus! Il se passe beaucoup de choses entre les paragraphes, qui ne sont pas dites et qu'il n'est pas nécessaire de dire non plus.

VoiX ET IMAGES La publication de Toute la vie a produit un bel émoi. Rarement a-t-on vu une couverture de presse comme celle qu'on a faite à votre livre. Quels sentiments en gardez-vous?

CLAIRE MARTIN J'ai trouvé étonnant de voir que je n'étais pas oubliée. Quand ce livre est sorti, on a su de qui il était. La couverture de presse évidemment a été merveilleuse.

voix ex IMAGES Durant la période de silence contenue entre Les morts et Toute la vie, avez-vous ressenti un manque? Un manque à exister, dans cette part de vous-même qui est disposée à l'écriture - à l'invention d'histoires où vous vous projetez, où vous vivez par procuration? Avez-vous éprouvé un manque à vous prolonger dans des existences fictives?

CLAIRE MARTIN Cela ne m'a sûrement pas créé un manque. D'abord parce que le travail a été remplacé par un vieux rêve que nous caressions, Roland et moi, depuis de nombreuses années. Et tout à coup, ce rêve se réalisait: nous sommes allés vivre là où on voulait vivre, sur la Côte d'Azur. Pendant ce temps, j'ai fait quelques traductions, c'est vrai. Mais ce travail n'aurait comblé qu'une partie du manque, s'il devait y en avoir un. $C^{\prime}$ est bien de traduire, mais on ne s'implique pas complètement, on n'est pas responsable de l'œuvre. L'œuvre est déjà faite. On va simplement tripatouiller dedans, comme ça. Et puis, un bon jour, Roland et moi, on s'est rendu compte que ce bonheur extrême qu'on goûtait, ce serait plus raisonnable de l'interrompre et de rentrer chez soi. De rentrer dans le raisonnable.

Voix ET IMAGES Vous vous êtes mariée peu après avoir annoncé sur les ondes la fin de la guerre. C'était en 1945. Entre votre dernier emploi, celui de présentatrice à RadioCanada, et votre premier livre, il s'est donc écoulé douze ans. Auriez-vous employé ces douze années principalement à lire et à parfaire vos connaissances?

CLAIRE MARTIN J'ai toujours été une lectrice fervente et constante. Et je lis encore beaucoup. Beaucoup plus lentement, par exemple. Parce que je n'ai plus cette hâte, cette curiosité; je m'arrête, je réfléchis, puis je relis un peu. Cela prend du temps. Je suis devenue un peu rêveuse dans mes lectures. Je m'arrête pour penser, si j'ai trouvé le passage beau. Des fois, c'est émouvant, alors on verse une petite larme. 
VoIX ET IMAGES Est-ce qu'il y a une famille d'esprit à laquelle vous estimez appartenir? De quel type d'écrivain ou de penseur vous sentez-vous plus proche?

CLAIRE MARTIN On reste proche de ce qui nous a atteint en premier. J'ai été très prise par les romanciers de l'entre-deux-guerres, qu'on recevait le plus fréquemment ici. Ce sont les seuls que nous ayons pu lire pendant la guerre, parce que de France il ne venait plus rien. Il en venait un peu de New York. La maison d'édition française qui publiait à New York les livres le faisait sans la permission des auteurs, qui étaient bien empêchés de donner leur permission, puisqu'ils étaient en France occupée. Et puis Bernard Valiquette à Montréal faisait la même chose, d'autres aussi. Il n'empêche que pendant la guerre, à part évidemment pour le fond de livres qu'on pouvait personnellement posséder, le choix était quand même limité. J'ai connu Roland en 1938 et il avait une assez bonne bibliothèque. À partir de 1939, il était comme tout le monde, il n'avait plus rien de nouveau. Mais tout ce qui nous arrivait, Les hommes de bonne volonté, en 27 volumes... j'ai lu tous ces livres avec la ferveur de qui n'a pas d'autre source nouvelle: les Pasquier de Georges Duhamel, Jean-Christophe de Romain Rolland. Il y en a sûrement d'autres que j'oublie. Les Salavin, de Georges Duhamel également. C'étaient de vrais romanciers, ces gens-là. C'étaient des romanciers qui pondent du roman et qui savent le faire. C'était leur vie! Un peu comme Troyat a fait ensuite. Troyat, à sa table de travail à 7 heures le matin. Il se lève pour dîner le soir. On vient lui porter un petit plateau de temps à autre. Je ne suis pas une lectrice de Troyat. Seulement j'admire qu'on puisse écrire avec cette obstination. C'est ça le vrai métier! Je me sens un peu comme la personne qui fait une chose, puis l'autre: l'amateur. Je me sens un peu comme un amateur.

VoIX ET IMAGES Il se trouve plusieurs écrivains français dont vous avez lu tout l'œuvre ou presque. Vous en avez nommé quelques-uns. Je pense à Proust, à Gide, à Colette et aussi à George Sand.

CLAIRE MARTIN Vous venez de nommer là des écrivains dont j'admire beaucoup la littérature, mais dont j'admire spécialement la correspondance. J'ai lu peu de romans de George Sand. Mais j'ai lu toute sa correspondance. C'est fascinant, vous savez, de voir cette femme qui écrivait jusqu'à quatre heures du matin. Elle commençait par écrire trois ou quatre lettres. Après elle écrivait du roman. Il faut dire qu'elle avait de grands besoins financiers parce qu'elle avait une fille qui lui était chère, puis un fils qui lui coûtait passablement d'argent. Puis une maison à Noyant. Quelle superbe maison! Ce n'est pas un château. C'est une grande maison. Belle et confortable. Des cuisines superbes. Il y a un système de chauffage central qu'elle avait inventé. C'était quelqu'un, madame Sand! Elle avait plein d'idées sur toutes sortes de choses. La correspondance de Gide est passionnante elle aussi. J'ai lu toute l'œuvre romanesque, les essais et le journal de Gide. Puis la correspondance avec Martin du Gard, avec Suarez, avec Claudel. Il faut lire les injures de Claudel: «Enfin monsieur, allez-vous avouer? En êtes-vous? Ou n'en êtes-vous pas?» Comme c'est grossier tout ça! Comment s'appelle-t-il ce poète avec qui Gide a fait une bringue assez poussée par moments, puis qui s'est converti? Le pauvre sous l'escalier, Henri Ghéon. Ils ont commencé par traîner ici et là tous les deux assez fréquemment. Tout à coup, Ghéon s'est converti. Comment dirais-je poliment? Il courait, mais pas dans le même sentier. Il s'est marié. Vous me direz que Gide s'est marié aussi, mais c'est une autre histoire, comme dirait Kipling. Alors la correspondance avec Henri Ghéon. La correspondance avec sa mère, ouf! C'est assez surprenant parce que la mère de Gide passait son temps, comme on dit vulgairement, à «chiquer la guenille». Elle n'était jamais contente. «Cela ne faisait pas. Ce n'était pas ci, ce n'était pas ça.» Elle le surveillait, mais 
elle n'a jamais rien compris. Malheureusement, la correspondance avec sa femme... Un jour sa femme s'est fâchée parce que Gide était parti pour l'Angleterre avec Marc Allégret. Elle a tout brûlé. Moralité? N'allez jamais en Angleterre avec...

voix ex IMAGES De George Sand, vous avez, bien sûr, lu Histoire de ma vie? Est-ce à vos yeux une autobiographie crédible?

CLAIRE MARTIN Parfaitement, c'est bien fait comme tout ce que fait Sand. Elle a choisi, je pense bien. Elle n'a pas tout dit. Personne n'est obligé de tout dire. Mais enfin, chez Sand, on se dit: «Oh, oh, oh! Madame, vous en passez de grands bouts.» Tout ce que Sand a fait, je trouve que c'est bien fait. Quoique, comme on dit maintenant - ça me vexe toujours un peu: "C'est dépassé». Si on se met à dire que c'est dépassé parce que ç'a été écrit il y a longtemps, à ce moment-là, qu'est-ce qui nous reste?

voix ET IMAGes Vous avez aussi mentionné Colette parmi vos écrivains de prédilection. C'est pour les mêmes qualités?

CLAIRE MARTIN Colette, ce n'est pas du tout la même chose. Colette est vraiment un écrivain de génie. Chose que j'apprécie beaucoup, elle est ce qu'on pourrait appeler un génie simple. Elle n'écrit pas des choses compliquées. Elle a un vocabulaire d'une grande beauté, des idées fraîches, des choses que personne n'a dites. Une façon habile de faire des romans. La naissance du jour, c'est son plus beau livre, qui est en fait moitié fiction, moitié réalité; tous ses personnages secondaires sont vrais. L'action se passe à SaintTropez, avant 1928. Tous ses personnages secondaires, tous les peintres qu'on voit, LucAlbert Moreau, et des écrivains, Paul Géraldy... Il faut savoir qui était Paul Géraldy, Toi et moi. C'est très curieux. C'est un livre de poésie amoureuse, petit comme ça, qui s'est vendu pendant cinquante ans, vendu partout, partout. Il en a tellement vendu qu'il a vécu richement, des grosses voitures, des Bentley. Des femmes avec des manteaux de zibeline. Pour en revenir à La naissance du jour... Il y a toute une histoire là-dedans. C'est un jeune homme de 35 ans qui est plus ou moins amoureux de Colette, qui voudrait être son amant, mais Colette dit que pour elle, c'est terminé l'amour. Maintenant, elle entre dans une vie d'ascèse. Et c'est juste au moment où elle venait de rencontrer Maurice Goudeket avec qui elle vivait une liaison passionnée, qui a fini par un mariage, d'ailleurs. Il a été l'homme de sa vie. Il a été là jusqu'au moment de sa mort. C'est comme quelqu'un qui voudrait conjurer le sort en écrivant une histoire où on est détaché de l'amour. D'ailleurs son mari avait 35 ans aussi, elle en avait 52. Elle n'est pas détachée du tout. Elle vit une grande passion. C'est très étrange. Il faut connaître la vie de Colette pour voir combien c'est étrange. Autrement, non. C'est surprenant, mais très bien écrit. Comme je pensais que vous alliez m'en parler, j'ai recopié quelques lignes sur le mistral. Ça me touche beaucoup, le mistral. J'ai bien connu ça et je voudrais vous le lire:

\footnotetext{
Retirée dans ma chambre j'attends avec une impatience modérée la retraite du visiteur, pour qui nulle vie n'éclôt. Et qui déjà pousse sous ma porte un singulier hommage de pétales flétris, de graines finement vannées, de sable, de papillons molestés. Va! J'ai découragé d'autres symboles et je n'ai plus quarante ans pour détourner le front devant une rose qui se fane. C'en serait donc fini de cette vie de militante.
}

Voix ET IMAGES Les romancières de la modernité, Virginia Woolf, Nathalie Sarraute, Marguerite Duras, les auteurs du Nouveau Roman, de la rupture avec la linéarité et la psychologie, est-ce qu'ils vous ont intéressée? 
CLAIRE MARTIN Marguerite Duras, non! C'est peut-être un peu de puritanisme chez moi. Je trouve que Marguerite Duras fait vicieuse, vous savez. Trop vicieuse! Ça me répugne! J'aime bien Nathalie Sarraute. Je n'ai pas lu tout Nathalie Sarraute, mais j'ai lu plusieurs de ses livres. Je l'ai rencontrée d'ailleurs à Ottawa et je l'ai trouvée une vieille dame aimable, qui disait des choses brillantes, qui était modeste en même temps. Tout ce qui me plaît chez quelqu'un qui a du talent. Une chose qui me plaît beaucoup, c'est l'œuvre de Virginia Woolf. C'est une œuvre triste. C'est talentueux. C'est une vie triste, aussi.

Voix ET IMAGES À la liste des auteurs que vous avez cités, on pourrait ajouter, je pense, La Fontaine, Flaubert, Stendhal ou Gracq...

CLAIRE MARTIN Gracq a été un des amours de ma vie. Gracq romancier. Les lettrines, c'est excellent aussi, mais il faut être en état de grâce pour le lire. Les romans de Gracq, Le rivage des Syrtes, Un balcon en forêt, Un beau ténébreux, ce sont des choses irremplaçables, pour moi. C'est quelque chose d'extrêmement bien fait et beau. C'est une littérature qui fait rêver, aussi. Cet entêtement dans Le rivage des Syrtes à guetter l'autre, à vouloir et s'en défendre et l'attaquer et finir par l'attaquer, que ça finisse dans le désastre, ça fait peur; et ça ouvre des vues sur des avenirs possibles.

VoIX ET IMAGES Outre certains livres qui auraient été déterminants pour l'écrivain que vous êtes devenu, les séjours à l'extérieur, parfois assez prolongés, ont-ils eu une importance aussi dans la maturation de votre talent?

CLAIRE MARTIN Quand on fait un voyage, même un peu long, un mois, deux mois, trois mois, c'est quand même du tourisme qu'on fait. On est beaucoup plus désireux de voir des choses que d'emmagasiner pour l'écriture. Mon principe, c'est que les livres viennent des livres. Pour écrire, il faut lire beaucoup.

Voix ex IMAGES De tous ces auteurs, s'il fallait n'apporter qu'un livre dans une île déserte, lequel choisiriez-vous?

CLAIRE MARTIN Je n'apporterais pas un livre d'auteur. J'apporterais un dictionnaire, parce que, dans un dictionnaire, il y a tout. Surtout un dictionnaire qui a une partie historique en plus d'un lexique. Alors, vous avez tout. On peut suivre des gens longuement, des généalogies, des familles. Si un dictionnaire est bien fait, il est plein de titres de livres aussi, avec des petits résumés. On peut se faire une idée des choses. L'étudiant disait à son professeur qui lui demandait: «Mais enfin, Monsieur, vous avez lu Stendhal?» Et il a répondu: «Pas directement.» Dans le dictionnaire vous pouvez tout lire, mais pas directement.

VoIX ET IMAGES Est-ce que vous attribuez un sexe à la voix de l'écrivain? Ou bien estimez-vous que la voix de l'écrivain participe des deux pôles, des deux entités, masculine et féminine?

CLAIRE MARTIN Je n'arrive pas à me faire une idée là-dessus. Je pense, évidemment, qu'il y a une façon d'écrire des livres qui est sûrement féminine. Je trouve que c'est une chose qui s'amenuise avec le temps. Des romans de femmes comme il y en avait au dixneuvième siècle, on n'en voit plus, je pense. Ce qu'on a à dire est bien différent, c'est sûr. Pour moi c'est une chose assez mystérieuse. Je ne vois pas très bien où se délimitent les sexes en littérature. Prenons Simone de Beauvoir. Ce qu'elle a écrit, c'étaient vraiment des livres de femmes. Je ne parle pas de la militante, je parle de la romancière. Sauf peut-être l'histoire de cet homme qui ne meurt jamais. Ce roman-là n'est pas un roman de femme.

VoIX ET IMAGES J'ai toujours cru que, pour commencer à écrire, il fallait s'être fait une idée du monde. Celle que vous vous êtes faite dans votre première jeunesse, sans doute 
l'avez-vous confrontée au système de pensée qui avait cours du temps où s'affirmait Simone de Beauvoir et alors que vous vous prépariez à écrire? Auriez-vous, par exemple, été gagnée par l'existentialisme, si présent dans l'après-guerre?

CLAIRE MARTIN Je me sens très étrangère à tous les «-ismes». Je n'aime pas les idéologies. Je n'aime pas les nationalismes. Je suis très patriote, mais je ne suis pas nationaliste. Je trouve que ce sont des choses désastreuses qui mènent à ce qu'on voit ici et là. Ça pousse toujours à tuer, ces choses-là. Il y a des gens qui ont dit que simplement le fait d'écrire, c'est déjà un engagement. Je veux bien. Je n'ai jamais eu d'engagement dans un parti ou dans une école. Ça ne m'intéresse pas du tout.

voix ET IMAGES Quand avez-vous su que vous alliez écrire des histoires? Étiez-vous par tempérament cette sorte de fillette imaginative qui invente des contes, des histoires pour ses frères et ses sœurs? Ou bien plutôt de celles qui se claquemurent pour lire, pour écrire leur journal intime?

CLAIRE MARTIN Je n'ai jamais écrit de journal intime. Je n'inventais pas de contes pour mes frères et mes sœurs, j'inventais des mensonges pour mon père. J'avais un grand talent pour cela. Une espèce de rapidité d'esprit qui faisait que je trouvais tout de suite. VoIX ET IMAGES Est-ce que le goût d'écrire, quand il est assez ferme et constant pour se réaliser dans des livres, appartient aussi à quelque chose d'inné?

CLAIRE MARTIN Je pense bien que ne sont écrivains que ceux qui sont nés écrivains. Autrement, ça ne sert à rien d'essayer. D'abord c'est un métier qui est dur, qui prend de la détermination, qui nous prive de beaucoup de choses. Il faut savoir se priver de tout cela avec plaisir et bonheur. Il faut aussi avoir un amour de la solitude. Et puis évidemment un amour de la langue, c'est l'enfance de la chose.

VoIX ET IMAGES Quelle est la faculté maîtresse d'un écrivain de fiction? Est-ce l'imagination, l'intuition, l'expérience du cœur et plus généralement des mœurs de l'anthropos, ce qu'on pourrait appeler, en fait, la culture générale? Ou bien serait-ce l'affinité avec les mots?

CLAIRE MARTIN On ne peut pas choisir dans tout ce que vous venez de dire là. Je pense que tout est nécessaire. Dans des proportions plus ou moins importantes. Il nous faut tout cela, comme l'affinité avec les mots, par exemple. Si cela nous manque, le texte va être plat, c'est sûr; ce qui n'empêche pas certaines personnes d'écrire. Il faut aussi une certaine expérience. On ne peut pas arriver à l'écriture avec naïveté. À moins d'écrire des livres pour enfants. Et encore! C'est peut-être ce qu'il y a de plus difficile à faire, des livres pour enfants. C'est pour ça que je n'en ai jamais fait, je pense bien. Un jour, on demandait à Michel Tournier: «Mais qu'est-ce que c'est un bon livre pour enfant?» Il a répondu: «C'est simple. C'est un bon livre pour adulte.» C'est probablement vrai. Pour la fiction, je pense que ce doit être d'abord l'imagination. Ça me semble absolument indispensable. Si on écrit une œuvre d'imagination, ce qu'on a imaginé doit appeler un certain vocabulaire. Chaque histoire pour moi comporte un certain nombre de mots clés que l'on doit conserver tout au long... Et puis évidemment la connaissance du cœur. Mais alors la connaissance du cœur? Si l'on fait du roman policier, on n'a pas beaucoup besoin de la connaissance du cœur. Connaître les mœurs, par exemple, alors c'est important. Et puis la culture générale, qui dépend évidemment de l'écrivain. Un écrivain peut quand même écrire des livres assez intéressants sans avoir beaucoup de culture générale. La culture générale, ça se prend dans les livres. Je pense qu'un bon écrivain a besoin de tout cela pour pouvoir écrire un bon livre, un livre excellent même. C'est rare d'avoir toutes les qualités. Je connais plus d'écrivains qui ont tous les défauts que ceux qui ont toutes les qualités. 
VoIX ET IMAGES Le style, sans doute, s'acquiert et se travaille. Mais il doit bien aussi tenir à une innéité puisque Buffon a pu dire cette chose que tout le monde cite: "Le style, c'est l'homme même.» Qu'est-ce que vous en pensez?

CLAIRE MARTIN Je pense que Buffon avait tout à fait raison. C'est quand même une chose qui s'acquiert et qui ne s'acquiert, à mon sens, que d'une seule façon. C'est dans les livres. C'est dans la lecture. Je passe mon temps à répéter que les livres viennent des livres. Si on ne lit pas, on ne peut pas être un bon écrivain. On ne peut même pas être un écrivain tout court. Quand j'entends quelqu'un qui fait métier d'écrire et qui me dit: «Oh moi, vous savez, je ne lis pas.» Je lui réponds: «Vous ne lisez pas? Mais enfin pourquoi écrire alors, si on ne lit pas?» Il me semble que, si on écrit, ce n'est pas simplement pour dire certaines choses à quoi on tient et dont on pense qu'elles sont les plus intéressantes du monde, $c^{\prime}$ est aussi pour s'insérer dans une tribu de gens qui écrivent. On s'engendre les uns les autres. On fait partie d'une filiation. Je ne vois pas qu'on puisse procéder autrement.

VoIX ET IMAGES Vous connaissez sûrement comme moi des conteurs extraordinaires, qui ont une verve, une faconde, une grande habileté avec la langue, des conteurs-nés qui tout à coup nous disent: «Je vais écrire!» On lit ce qu'ils ont écrit et on trouve cela désolant. Ça vous est déjà arrivé?

CLAIRE MARTin Cela m'est arrivé. Et dans les Pasquier de Georges Duhamel, il y a le père, qui est un homme fabuleux et qui raconte des histoires inouïes. Un jour, il décide d'écrire un roman. Et c'est d'une tristesse... ! tellement c'est plat. Ce sont deux choses bien différentes, de pouvoir écrire et de pouvoir parler, me semble-t-il. Un de nos grands écrivains, Alain Grandbois, dans l'intimité, ne disait que des platitudes.

VoIX ET IMAGES Tous les commentateurs soulignent la concision de votre écriture, la quête du trait essentiel, révélateur et efficace, d'autant plus qu'il est unique. Cette recherche, je pense, est fondamentale pour vous. Je me rappelle vous avoir entendu citer: «Qui ne sait se restreindre ne sut jamais écrire.»

CLAIRE MARTIN Du moment qu'on sent que c'est délayé, qu'on tire à la ligne, comme on disait du temps où tous les grands écrivains publiaient en feuilleton, on sent tout de suite le côté pénible.

voiX ET IMAGES Outre la concision, il semble que la simplicité aussi fasse partie des qualités que cultive votre style.

CLAIRE MARTIN La simplicité est très difficile à acquérir. J'aime bien tarabiscoter un peu de temps en temps. Pour faire ressortir la simplicité un peu plus loin. Comme j'aime bien faire des phrases courtes, puis tout à coup faire tout un paragraphe de douze lignes, où il y a des virgules, mais pas de point. Je ne ferais pas ça bien longtemps, par exemple, parce que je pense que la respiration dans un livre est importante. La simplicité d'ailleurs fait partie de cela. Une phrase qui est longue, qui comprend beaucoup d'incises, ce n'est pas simple, tandis que, si vous avez six mots, $c^{\prime}$ est bien difficile de ne pas comprendre. Voltaire a dit: "Le génie de la langue française, c'est la clarté et l'ordre.» J'y tiens beaucoup. À ce qu'il y ait de l'ordre et qu'il y ait de la clarté! Et que la clarté soit aussi dans l'harmonie des phrases, l'harmonie des mots qui se succèdent les uns les autres. Si c'est laid, si l'oreille n'est pas contente, s'il y a une insatisfaction, c'est que ce n'est pas bon. VoIX ET IMAGES Je sais que vous attachez beaucoup d'importance à la précision de la facture. C'est à La Bruyère qu'il faut attribuer les paroles souvent citées: "C'est un métier que de faire un livre comme de faire une pendule. Il faut plus que de l'esprit pour être auteur.» Vous qui aimez coudre et cuisiner, vous devez être sensible à cette opinion, au tour artisanal que peut prendre l'élaboration d'un livre. 
CLAIRE MARTIN Sûrement! C'est pour cela que j'écris. Il y a dans l'écriture un côté manuel qui m'est indispensable. J'aime travailler de mes mains, j'aime faire mes choses. Par exemple, j'aime cuisiner, j'aime recevoir. Tout ça fait le caractère d'une personne qui fabrique des personnages qui, eux, font toutes sortes de choses, qui sont capables de vivre de façon autonome. C'est très important pour moi. Je ne sais pas à quel propos, je me disais: "Tiens, c'est la même chose quand on veut élaborer une œuvre manuelle.» Il faut de l'habileté, il faut savoir où on va. Je pense que c'est un peu pareil quand on écrit. J'écris à la main, d'abord. Je trouve ça très précieux parce que si, tout à coup, j'écris un mot que je viens d'employer sept ou huit lignes plus haut, je le sens tout de suite. J'ai horreur des répétitions, à moins qu'elles ne soient nécessaires, voulues. Alors, c'est une répétition qui devient une figure de style.

VOIX ET IMAGES «Dans tout écrivain, il y a un grammairien» (je cite très approximativement Victor Hugo). S'agit-il d'une proposition susceptible de vous rallier?

CLAIRE MARTIN La grammaire, c'est une de mes passions. La grammaire, c'est la civilisation. C'est ce qui met la parole en forme et ce qui fait que nous sommes des êtres qui parlent, qui pensent, qui s'expriment. Avant l'arrivée de la civilisation, ça n'existait pas. Personne ne savait ce que c'était que la grammaire, personne n'en avait besoin non plus. La grammaire, c'est l'affirmation même de la civilisation.

VoIX ET IMAGES Selon vous, la connaissance fine de la langue serait donc un domaine de responsabilité pour un écrivain?

CLAIRE MARTIN Nous avons valeur d'exemple. C'est la défense et l'illustration. Si on essaie de défendre et d'illustrer autre chose, pour moi c'est une mauvaise action. Je me suis souvent exprimée là-dessus.

VOIX ET IMAGES Autrefois, pour baliser un usage qui est toujours changeant, on s'en remettait au parler des Halles. On dit qu'à présent la règle se constitue à partir des livres, des publications. C'est donc que l'écrivain, le journaliste auraient un rôle dans l'évolution de la langue. Considérez-vous, comme le philosophe Claude Lévesque, que l'une des fonctions de l'écrivain est de renouveler et d'élargir la langue?

CLAIRE MARTIN En fait, la responsabilité de l'écrivain dans la langue est quelque chose d'inéluctable. Est-ce que tous les écrivains vont dans le sens de l'amélioration, du perfectionnement? Je ne le crois pas. Mais ce devrait être le rôle de l'écrivain vis-à-vis de la langue d'en faire quelque chose qui soit toujours plus nécessaire, plus beau, plus utile. Bien souvent, ce n'est pas le cas. Maintenant l'écrivain a beaucoup de rivaux: la télévision, la radio, les journaux.

voIX ET IMAGES Dans les livres qui consignent l'usage, comme les dictionnaires, par exemple, on cite des écrivains d'un peu toutes les époques, surtout du dix-neuvième et du vingtième siècles. Toutes sortes de mots s'ajoutent dans la langue. Est-ce que vous, comme écrivain, vous vous donneriez la liberté d'inventer des mots et de proposer des usages nouveaux, de faire des jeux syntaxiques qui ne sont pas encore codifiés mais que vous savez exister par exemple dans la parole?

CLAIRE MARTIN Cela m'est arrivé souvent de me dire que le mot que je voudrais, il faudrait que je l'invente puisqu'il n'existe pas. J'ai beau chercher, il n'y a pas de mot avec ce sens précis. Ce serait très difficile de faire admettre... Quelqu'un va mettre sic en me citant... VoIX ET IMAGES Montaigne et Saint-Simon ont travaillé à faire passer l'usage parlé dans l'écrit. Barbusse, Queneau, Ducharme incorporent les inventions du discours à leur langue. Quand elle est dans le milieu du music-hall, Colette en reproduit la langue, qui est très colorée et très révélatrice des mentalités de ce milieu. 
CLAIRE MARTIN Proust le fait dans d'autres contextes. Il peut être très drôle. Il employait, un temps, le mot «louchonnerie». «Oh! C'est de la louchonnerie...» Cela avait l'air d'être un terme qu'ils employaient entre amis, qu'ils avaient inventé pour je ne sais quel usage. Qui voulait dire qu'on rit tellement, que c'est tellement ridicule, qu'on finit par en loucher. Le mot revient souvent. Proust est plein de surprises. Ses amis, quand ils parlent de lui, nous étonnent: on a toujours vu Proust comme un être maladif et sans force, faiblard. C'est Roger Stéphane qui raconte que Paul Morand lui a dit qu'un jour Proust avait son taxi qui l'attendait. Il monte dans son taxi - c'était avant 1918 - il y avait un militaire américain qui était monté par l'autre côté. Vous savez, un militaire américain, quand même! Proust l'a poussé dehors, puis il est parti aux applaudissements des badauds qui étaient sur les trottoirs.

VoIX ET IMAGES Avez-vous ce genre d'insécurité qui vous pousse à consulter le dictionnaire constamment lorsque vous écrivez?

CLAIRE MARTIN Pas quand j'écris ce que j'appellerai mon premier jet, mais après, oui. Il faut tellement être sûr qu'on en arrive à ne plus être sûr de rien. On dit deux «l» ou un seul? On sait bien que c'est deux, mais on va voir. On en perd du temps comme ça!

voix ET IMAGES Dans le débat sur la langue, les prises de position se concentrent toujours autour de deux pôles. Il y a celui des linguistes et des historiens de la langue, qui sont plus détachés, plus tolérants, qui expliquent, études et enquêtes en main, qui justifient même les écarts. Et puis il y a celui des puristes, qui s'enflamment, qui dénoncent, qui ne veulent pour mesure de correction que la norme de l'Académie française. Où vous situez-vous, vous que la langue ne laisse pas indifférente?

CLAIRE MARTIN Je ne me situe pas dans le premier groupe, bien sûr, ni dans le deuxième. Je ne suis pas braquée sur les ukases de l'Académie française. Mais je n'accepterai jamais de dire que notre langue est un français à part, le français québécois. Nous sommes des francophones. «Ah! vous voulez écrire comme les Français!» Ma langue, $c^{\prime}$ est le français parce que je suis francophone et que je voudrais que tous les francophones du monde parlent le mieux possible et non pas le plus mal possible. Il y a des gens qui croient qu'il y a seulement une chose qui est faite pour nous et c'est ce qu'il y a de plus laid. Cette humilité-là ne m'atteint pas du tout. J'aime bien l'humilité, mais pas celle-là. Ou l'air de dire que le vrai français, c'est trop difficile pour nous. Je pense qu'ici nous sommes plus nombreux à mal parler. Je me fais encore dire parfois: «Mais pourquoi vous parlez comme ça?» Parce que c'est ma façon de parler. Qu'est-ce que vous voulez? Je ne peux pas en changer. J'ai toujours parlé comme ça. Ou bien des gens croient que je parle ainsi parce que j'ai vécu en France. Je parlais comme ça avant d'aller vivre en France. Dans ma famille, on était très exigeant sur la façon de parler.

VoIX ET IMAGES Lorsque vous avez fait paraître vos mémoires, la tendance était, parmi les jeunes intellectuels au moins, à revendiquer la transmission directe dans l'écrit de la langue parlée. Le modèle choisi, pour les raisons politiques qu'on connaît, était celui du prolétariat montréalais. Avez-vous subi des pressions de vos collègues ou du public pour vous attacher à cette pratique?

CLAIRE MARTIN Pas au moment où j'ai écrit. Parce qu'au moment où j'ai écrit Dans un gant de fer et La joue droite, je le faisais dans mon quant-à-soi. Par la suite, j'ai subi des pressions. Il y a même des gens qui m'ont dit des choses assez injurieuses : j'étais «traître à mon pays! À ma langue!» Cela me semble ressortir à cette chose énorme et très forte, dont on doit se tenir éloigné : la bêtise humaine. Les jeunes intellectuels y étaient très portés, c'est vrai. Ce que je trouve triste là-dedans, c'est qu'on puisse 
appeler «intellectuels» les tenants d'une telle attitude. Pour moi, intellectuel, ce n'est pas une injure. Traiter quelqu'un d'intellectuel, c'est un grand compliment. Voir les intellectuels qui cherchent de façon tout à fait artificielle à écrire le plus mal possible, $c^{\prime}$ est un mystère. Mais ce n'est pas le genre de mystère que j'aime.

voix ET IMAGES Dans votre langue écrite, vous seriez-vous permis d'utiliser des canadianismes, des régionalismes, mettons, «de bon aloi»?

CLAIRE MARTIN Si j'avais écrit quelque chose dans ce sens-là, bien sûr. Si j'avais dû citer à un moment un dialogue vrai où quelqu'un eût parlé de cette façon, oui. Dans un passage de Dans un gant de fer, j'ai fait dire une phrase à la vieille bonne, chez nous, et je lui ai fait parler son langage, à ce moment-là.

VoIX ET IMAGES On a beaucoup parlé de votre connaissance du cœur et du thème de l'amour qui est récurrent dans votre œuvre et qui est en effet traité avec beaucoup de subtilité. Est-ce que vous vous seriez mise tardivement à écrire pour justement acquérir d'abord cette expérience du cœur?

CLAIRE MARTIN Tant que je n'ai pas commencé à écrire, si je n'écrivais pas, c'est parce que je ne me sentais pas prête à le faire. Je n'avais pas l'intention de commencer par un livre dont on aurait dit: «Enfin, ça ne vaut pas grand-chose, mais c'est un premier livre. Elle fera mieux la prochaine fois.» Je voulais faire quelque chose qui avait, à mes yeux en tout cas, un certain achèvement. Quand ça m'est arrivé, j'avais 44 ans. Ce n'est pas l'extrême jeunesse! Cela me faisait bien rire quand je suis allée pour le lancement d'Avec ou sans amour à Paris. Dans tous les journaux, on disait «la Françoise Sagan québécoise». Mince de Sagan, alors! Françoise Sagan a écrit ce qu'elle a écrit parce qu'elle a commencé très jeune. La tristesse de l'adolescence. Ce n'était pas une chose que j'aurais voulu faire, que j'aurais pu faire.

Voix ET IMAGES Dans votre entreprise d'écriture, quelle est la part dionysiaque et quelle est la part apollinienne?

CLAIRE MARTIN Je suis très apollinienne. À chaque fois que j'ai écrit un roman, j'ai suivi une direction bien nette, bien établie à l'avance. Je savais où j'allais. Je savais comment ça commencerait, comment ça se continuerait, comment ça se terminerait. Je ne suis pas de ces écrivains qui disent que leurs personnages les mènent. Il faut savoir où l'on s'en va. Quand ça va dans toutes les directions, quand c'est fumeux par moments, on se dit: "Ça n'a pas été pensé». Or un livre, il faut que ce soit pensé.

VoIX ET IMAGES On pourrait imaginer, par exemple, que vous êtes prise d'inspiration et que vous faites un premier jet de cette façon un peu tumultueuse et qu'ensuite une seconde écriture et même, pourquoi pas, une troisième écriture met en place les choses qui ont un peu erré?

CLAIRE MARTIN Cela m'est arrivé, non pas dans la fiction, mais quand j'ai écrit Dans un gant de fer et La joue droite. Il m'est arrivé d'enlever des pages complètes en disant: «Non, cela n'apporte rien de plus; c'est parfois trop désagréable; puis c'est parfois trop douloureux, c'est parfois trop ridicule». Autrement, ça ne m'est pas beaucoup arrivé, sauf dans Les morts, qui est une conversation. Et la conversation, même dans un livre, même une conversation tout à fait artificielle, celle que l'on imagine pouvant devenir un livre, porte un peu à se laisser aller à tenir toutes sortes de propos adventices qui ne sont pas absolument nécessaires. Et pour se faire plaisir. Quand on a fini de se faire plaisir, souvent, on met à la poubelle.

Voix et IMAGES Est-ce que la complaisance est un des défauts, un des écueils de l'écriture? 
CLAIRE MARTIN On sent très bien cela quand on lit quelqu'un qui se laisse aller à la complaisance. On dit: «Tiens! Il aurait pu enlever ça, ce n'était pas tout à fait nécessaire». Et puis, ça fait un peu risible ; ou ça nuit à la compréhension. Tout cela fait partie du manque de rigueur.

voiX ET IMAGES Sauf dans l'exemple que vous avez donné, ce n'est pas un procédé qui vous est familier que celui de la soustraction, d'avoir écrit emportée par un mouvement, d'avoir beaucoup écrit pour ensuite soustraire. Mais cela ne vous est-il pas arrivé pour un roman comme Doux-amer? Qui peut sembler proche de l'autobiographie?

CLAIRE MARTIN Dans Doux-amer, il n'y a pas eu beaucoup de ratures, d'ajouts, de soustractions. Je savais vraiment ce que je voulais faire et où je voulais aller. D'autant plus que, pour moi, Doux-amer c'est ce que j'appellerais un roman-roman. Cela n'a rien d'autobiographique. Je ne parle pas des sentiments, de la façon de penser. Mais dans l'intrigue, il n'y a absolument rien d'autobiographique. Mes personnages ne me ressemblent pas. Gabrielle est un écrivain qui a écrit toute sa vie, qui n'a jamais cessé d'écrire, qui était extrêmement malheureuse quand elle ne pouvait pas écrire. Moi, j'ai abandonné l'écriture en 1972 avec un bonheur extrême. J'ai bien fait, parce que, quand je l'ai retrouvée, c'est avec le même bonheur. J'ai retrouvé mon bonheur, là, qui m'attendait. Et Gabrielle est une femme à foucades, en plus, parce que de temps en temps l'esclavage du métier fait qu'on se jette tout à coup dans des choses incompréhensibles. Je ne suis pas du tout ce genre de femme. Et puis l'éditeur ne me ressemble pas non plus. Il me ressemblerait peut-être un peu plus que Gabrielle. Il est plus rassis, plus attentif aux choses, plus rêveur.

VoIX ET IMAGes Pourquoi tenez-vous à ce point à vous distancier de Gabrielle? Et pourquoi tenez-vous toujours à affirmer qu'il n'y a rien d'autobiographique dans ce roman?

CLAIRE MARTIN Parce que la plupart du temps, quand on en parle, on dit: «Bon, évidemment, on sent ce n'est pas pour rien qu'elle a choisi un écrivain.» Aussi parce qu'on revient toujours à dire qu'ordinairement le premier livre d'un écrivain est toujours autobiographique. Qu'est-ce que cela fait que ce soit autobiographique ou non? Ça me surprend toujours quand on me le dit, parce que je ne me reconnais absolument pas dans Gabrielle.

VoIX ET IMAGES Quand vous êtes au point de commencer une histoire, avez-vous véritablement tracé des plans sur des feuillets? Avez-vous écrit des notes concernant les personnages? Avez-vous l'idée d'un développement détaillé? Est-ce qu'il y a des esquisses, une préfiguration écrite de ce que sera l'œuvre?

CLAIRE MARTIN Oui, pour Doux-amer et pour Quand j'aurai payé ton visage. Pour Les morts, ce n'est pas du tout la même façon de procéder. J'avais une ligne directrice; je m'étais beaucoup attachée à la façon dont parlent deux personnes, à la manière dont on mène une conversation. C'est d'ailleurs pour ça, par défaut de plan, que j'ai dû jeter beaucoup.

voix ET IMAGES Les débuts, c'est toujours ce qu'il y a de plus difficile. Et les fins sont difficiles à imaginer. Pas pour vous?

CLAIRE MARTIN La fin, ça m'arrive comme ça, comme un don, ordinairement. La dernière phrase, la chute, surtout pour les nouvelles, tout à coup, pouf! Ça vient comme un œuf. Dans les romans c'est un petit peu plus amené, bien sûr. Pour moi, le premier paragraphe est le plus important. Si j'ai mon premier paragraphe et s'il est bon, ça peut filer. Je ne comprends pas comment un écrivain ose nous offrir un livre où le premier paragraphe n'est pas passionnant. 
VoIX ET IMAGES La narration confiée au «je» est de loin la plus fréquente dans votre œuvre. Correspond-elle au caractère d'intimité et d'analyse de votre fiction, à une recherche de vraisemblance par l'effet de proximité?

CLAIRE MARTIN Oui, mais aussi à une sorte de nécessité chez moi, d'écrire à la première personne. Si bien que le paragraphe initial, il m'est arrivé d'essayer de le mettre à la troisième personne. Et c'est devenu confus. Je vois que je ne me dirige bien que dans le récit à la première personne.

VoIX ET IMAGES Et au fond, pourquoi avoir choisi la fiction?

CLAIRE MARTIN Pour beaucoup de gens, la fiction sert à cacher la vérité. Pas pour moi. Elle correspond sûrement à un besoin d'écrire une histoire, telle histoire et non pas telle autre. Mais d'où vient ce besoin? Et quel besoin pour l'autre de lire ce qu'on a écrit? Comme c'est mystérieux, tout cela! Il y a des gens comme ça qui, depuis Homère, ont besoin de raconter. Ils ont des choses à dire. Ils ont imaginé quelque chose dans leur tête, puis il faut le dire à quelqu'un.

Voix ET IMAGES Lorsque vous entreprenez un récit, une nouvelle, un roman, est-ce que le récit est déjà potentiellement chargé d'une intention morale, ou bien l'intrigue se suffitelle à elle-même?

CLAIRE MARTIN Je suis assez moraliste, finalement. J'ai l'air d'une vieille scélérate, comme ça, mais je suis pleine de bons sentiments. Je pense que c'est à cela que mes récits en viennent habituellement. À ce que les choses se placent dans une certaine moralité, qui n'est pas toujours la moralité admise par tout le monde. Certain état des choses qui fait que tout est bien - tout n'est peut-être pas romanesque, mais tout est bien. C'est ainsi que finissent mes romans habituellement.

voix ET IMAges Quand l'idée des Morts vous est venue, c'était parce que vous étiez présente à ce thème de la mort ou bien parce que vous vouliez traiter de la pérennité de l'amitié au-delà de la mort? Ou de l'amour au-delà de la disparition des êtres élus?

CLAIRE MARTIN Il y a sûrement la question de pérennité, qui est symbolisée par le fait que ces morts ont toujours le même nom, ils s'appellent toujours de la même façon. En somme, ils devraient s'appeler André parce que André veut dire l'homme. Il y a une pérennité à s'appeler André. On attire tous les hommes derrière soi. J'avais toutes sortes de choses que je voulais établir. Je ne sais pas si c'est bien ressorti dans le livre. C'est que finalement on n'aime qu'une fois. Il peut y avoir des choses avant ou après l'arrivée du vrai amour, mais ça ne compte pas vraiment. On doit peut-être aussi, quand il y a plusieurs personnes dans la vie de quelqu'un, chercher à peu près la même personne dans tout le monde. Puis il y a aussi l'idée de la mort qui n'est pas tellement importante là-dedans. Ça s'appelle Les morts, mais ça veut surtout dire les disparus. C'est un livre aussi contre la guerre. Et contre l'honneur de mourir chez les hommes.

voix ET IMAGES Le thème de la mort lui-même, vous ne l'avez donc jamais traité. Ce serait dans les projets que vous caressez?

CLAIRE MARTIN Peut-être l'ai-je fait dans les premières nouvelles... Non, parler vraiment de la mort, je n'en ai pas envie. D'abord, ce n'est pas bien intéressant, quand on y pense. Ce n'est pas que je trouve ça horrible ou désespérant. Pourquoi la mort fait-elle tellement peur? Je n'ai pas peur de la mort. Je la vois d'une façon assez paisible, parce que je n'ai pas du tout été imbibée par cette notion que ce qui peut nous attendre après la mort est quelque chose de peut-être horrible. Pour moi, après la mort, il n'y a rien du tout. Quand on en est bien persuadé, on envisage la mort avec calme. 
VoIX ET IMAGES Vous rappelez-vous Ionesco? Lorsqu'il s'est senti malade et proche de la mort, il a fait des efforts pour essayer de croire, pour justement ne pas arriver à l'idée de la disparition totale. Vous n'êtes pas tentée, vous, par des croyances qui vous assureraient une transfiguration de vous-même dans une autre énergie, ou une renaissance, un éternel retour?

CLAIRE MARTIN Je sais bien que mes cellules ne mourront jamais. Sur notre planète rien ne se crée, rien ne se perd. Je suis faite de choses qui ont déjà existé. Je ne sais pas si ce sont des choses ou des personnes, et puis ça restera. Pour moi, c'est ça, l'éternité.

voix ex IMAGES Parlons un peu de votre manière de travailler. C'est l'après-midi, je crois, que vous consacrez à l'écriture? Vous conformez-vous à un horaire?

CLAIRE MARTIN Penser «horaire», c'est penser à des heures, à plusieurs heures j'imagine. Je fais seulement une demi-heure, parfois deux heures. Mais toujours l'après-midi.

voIX ET IMAGES Quelle place accordez-vous à l'écriture dans votre vie?

CLAIRE MARTIN La place normale que l'on accorde au travail. C'est mon travail.

VoIX ET IMAGES Et comment se constitue la matière de ce que vous allez écrire?

CLAIRE MARTIN C'est comme toute création: on ne sait pas trop trop d'où ça vient. Parfois il y a des sujets que j'ai gardés derrière la tête pendant des années, comme par exemple celui de L'amour impuni. C'est une chose que j'avais envie d'écrire depuis très longtemps. Mais je n'avais pas trouvé le ton que je cherchais. Puis tout à coup, je l'ai trouvé. C'est un ton sympathique. J'aurais été portée au tout début à employer un ton ironique. Je me suis dit: de quel droit employer l'ironie pour ça? Finalement, ce que j'ai écrit, c'est exactement ce que je voulais. C'est une sorte de conte de fées.

VoIX ET IMAGES Est-ce que vous imaginez des antécédents à vos personnages?

CLAIRE MARTIN Bien sûr! Voilà ce qui ne cesse de me frapper: les antécédents et surtout les petits faits de la vie expliquent le personnage. Par exemple, dans La brigande, il y a toutes sortes d'événements qui ne vont pas plus loin que la mention. Vincent, il apparaît, on ne le retrouve plus? mais non! Il est là pour mettre en lumière un trait du caractère de Cora, c'est tout. Et puis, elle, elle a une sœur qu'elle aime beaucoup. Je pense que cette relation sert à faire ressortir le caractère chaleureux de mon personnage, qui n'est pas occupé seulement de sa peine d'amitié, de ses amours anciennes, et de ses amours nouvelles. Elle a aussi, on en prend connaissance dans l'action, un amour fraternel. (Une chose extrêmement importante dans ma vie, l'amour fraternel.)

VoIX ET IMAGES Lors de la publication de Toute la vie, il semblait peu probable que vous reveniez jamais au roman. Vous vous disiez désireuse de continuer à écrire, mais plutôt des œuvres courtes. L'amour impuni est-il donc une nouvelle qui aurait tourné au roman?

CLAIRE MARTIN C'est un roman que j'aurais pu faire plus long, mais je ne veux plus faire des choses longues. J'ai envie de faire le plus ramassé possible. Pas d'explications, les faits bruts. Ils sont là pour qu'on sache évidemment où l'on va, puis pour qu'on devine aussi à qui on a affaire. J'ai écrit Toute la vie avec beaucoup de plaisir; enfin ce que j'en ai écrit à l'heure où le livre a été publié. Mais après, je me suis dit que c'était difficile d'écrire un recueil de nouvelles parce que ça prend une idée neuve pour chaque pièce. En fait, un roman convient plus à mon désir de travail.

VoIX ET IMAGES Au moment où vous vous disiez: non, plus de roman, est-ce parce que le roman vous paraissait manquer de pertinence de nos jours?

CLAIRE MARTIN Je ne crois pas. Je crois à la survivance éternelle du roman. Il y a quelque temps, dans Propos sur le bonheur d'Alain, j'ai lu avec beaucoup de plaisir: «Pour moi, la 
vraie littérature, c'est le roman. Je ne lis que des romans.» Or c'était quelqu'un quand même, Alain. On peut se fier à son opinion! Je me suis dit: la vraie littérature, c'est le roman. En fin de compte, l'ensemble du lectorat, comme on dit maintenant, pense ainsi. Évidemment, il y a des essais fulgurants qui paraissent. Mais ce qui retient l'attention générale, c'est le roman.

voIX ET IMAGES Que dites-vous de l'affirmation répandue qu'un écrivain n'écrit jamais que la même œuvre sous différentes formes?

CLAIRE MARTIN Je crois que c'est vrai. Chez moi, c'est une chose et son contraire. Mes titres sont toujours comme ça aussi: Avec ou sans amour, Doux-amer... Je pense que c'est assez important dans ma façon de voir les choses, de les établir dans un livre. Jusqu'à quel point cela correspond-il à ce qu'on est? Je me rends compte que, dans mes premiers livres, il y avait déjà une espèce d'antagonisme entre l'homme et la femme; une espèce de rancune. Pourtant ce n'est pas du tout ce que je vivais dans ma vie personnelle. Je me demande maintenant si cet antagonisme n'était pas simplement ce que je ressentais vis-à-vis de mon père et des hommes de sa génération; ce dont j'ai beaucoup souffert.

VoIX ET IMAGES Les commentaires et les critiques ne vous ont-ils jamais révélé des aspects de vous, de vos personnages, de votre manière, que vous ignoriez?

CLAIRE MARTIN Je pense qu'on écrit et qu'on ne se rend pas bien compte si c'est comme ceci ou comme cela, ou si cela tient à telle façon d'écrire, à telle école... On ne peut pas avoir le même regard que les autres sur ce qu'on fait. Ce n'est pas possible. Enfin, c'est inexplicable! Et je trouve que tout ce que je lis sur ce que j'ai fait m'étonne.

VoIX ET IMAGES L'amour impuni et La brigande se rattachent tous deux au thème si fécond dans votre œuvre de l'affectivité positive. La poursuite du bonheur, sa réalisation constituent-elles à vos yeux le but de l'existence?

CLAIRE MARTIN C'est sûrement un des buts de l'existence; c'est probablement le motif caché que l'on a, motif secret, même secret à soi-même. Je ne crois pas que le contraire puisse être vrai. Il faut être un peu fou pour rechercher le malheur. Pour moi la quête du bonheur est une chose très importante. Le bonheur, on se le doit à soi-même. Quand on est heureux, c'est là qu'on donne le meilleur de soi. C'est quelque chose qu'on doit à son entourage aussi. C'est un acte de charité que d'être heureux. Ça déteint sur tout l'entourage.

Voix ET IMAGES On constate à vous lire que l'intérêt de la romancière loge dans l'évolution du sentiment vers le calme et le bonheur tendre plus que dans la crise passionnelle. Cela correspond-il à une conviction que vous assumez? L'amour trouve-t-il sa juste, sa vraie affirmation dans la continuité?

CLAIRE MARTIN Je ne conçois pas qu'un véritable amour ne soit pas durable. Malgré tout ce qu'il peut traverser. Je pense que c'est dans la durée et dans l'espèce de calme qui vient après les orages du début. La passion physique, même si elle reste, ne sera plus cette chose folle qu'on connaît au début de l'amour; ce sera devenu une aimable habitude. Ce n'est pas à rejeter non plus. Je ne conçois pas l'amour autrement.

VOIX ET IMAGES Le titre L'amour impuni pourrait faire entendre que la réunion des amants s'atteint par-delà une transgression et que leur bonheur est un défi. Cette intention existe-t-elle? S'agit-il d'un amour condamné échappant par miracle au désastre? Ou bien tout amour risque-t-il sanction?

CLAIRE MARTIN Presque toutes les amours sont condamnées à s'éteindre à un moment donné. Dans le cas de L'amour impuni, je l'ai intitulé ainsi parce que la plupart des amis 
homosexuels que j'ai eus n'étaient pas heureux dans leurs amours. Pas très longtemps en tout cas. Ou bien même j'en ai connu qui n'avaient pas d'amour. On attire beaucoup les confidences quand on est romancier. Des gens m'ont dit: "Je n'ai rien dans ma vie. Ça ne marche jamais. Je n'ai personne.» Comment se fait-il qu'ils s'aiment encore dans mon livre? Parce que c'est un livre. Je ne sais pas comment expliquer. Il y a aussi les menaces, elles étaient très présentes dans le temps de l'écriture. Maintenant, on en parle moins. Elles existent pourtant toujours, les menaces concernant la santé. Certains homosexuels ont vécu dans la peur. Ce n'est pas bon pour l'amour d'exister dans la peur. On leur connaît aussi une mentalité un peu différente de celle de l'hétérosexuel. C'est que l'homosexuel est souvent un peu coureur, et même beaucoup coureur. Dans ce cas, ça n'a rien à voir avec l'amour.

VoIX ET IMAGES Le contenu au passé des existences est toujours difficile à manœuvrer, à insérer dans l'action. Généralement, vous vous arrangez pour ne pas le faire, pour ne pas avoir de retour en arrière.

CLAIRE MARTIN Dans La brigande, on est quand même tout le temps plongé dans le passé, par les lettres, pas très explicites, en fait. Tout simplement, elles indiquent le caractère pervers de Nicette. Elles sont simplement là pour montrer comme Nicette ne méritait pas d'être aimée.

VoIX ET IMAGES Il est question dans La brigande d'un manuscrit perdu puis retrouvé. Je me rappelle, alors que vous songiez seulement à reprendre la plume, vous avoir vu tentée par ce motif. Est-ce ainsi que vous pensiez lui donner conséquence?

CLAIRE MARTIN Ce n'est pas du tout ce que je voulais faire. Ce que je voulais faire, je l'ai commencé, je l'ai abandonné. Ç'aurait été un manuscrit ancien qui aurait été volé pour des fins de collection sûrement. Tout à coup, ça s'est mis à me sembler rébarbatif. J'ai été prise par mes obligations, je l'ai perdu de vue pendant un certain temps. Quand je l'ai retrouvé, je me suis dit que je ne voulais plus de cette histoire-là. Mais l'affaire du manuscrit perdu, c'est une chose qui m'a toujours hantée. Je n'en ai jamais perdu mais j'ai toujours tremblé que ça m'arrive. C'est une affaire épouvantable de perdre un manuscrit.

VoIX ET IMAGES Et c'est ce qui arrive à Cora, la narratrice de La brigande. Elle n'en parle pourtant pas sur un ton déchirant. Non plus qu'elle ne le fait de ses déceptions sentimentales.

CLAIRE MARTIN Les critiques consacrés ont dit que c'était un livre léger. Ça m'a soufflée. Un livre léger? Le ton est léger? Il faut savoir dissocier le ton du reste. J'emploie toujours un langage retenu. Ça fait mille fois que je le répète, mais je ne le dirai jamais trop. J'ai toujours été d'accord avec Proust quand il dit: «N'expliquez pas! Ne dites pas tout!» On lit quelque chose, suit une explication. On avait compris. Ou bien: on ne voulait pas le savoir! Je me tiens toujours un peu sur la défensive, pour ne pas en dire plus qu'il n'est absolument nécessaire.

voix ET IMAGES Le sentiment de Nicette pour Cora est assez trouble. Et vous ne l'éclairez pas mieux qu'avec la découverte du manuscrit perdu que Nicette avait peut-être entrevu de faire paraître sous son propre nom. Il nous semble alors voir se profiler un cas de cannibalisme. La défection apparente à l'amitié devient quelque chose d'autre. Une rage d'appropriation plutôt, qui tâche d'isoler l'être qu'elle jalouse de tous ses bonheurs et dont elle irait jusqu'à s'approprier la personnalité comme s'il ne s'agissait au fond ni de détestation ni de haine, mais de l'envers d'une amitié incapable d'exprimer une pareille exigence. Serait-ce la face destructrice de l'amour? 
CLAIRE MARTIN Je me suis souvent posé la question. J'ai connu des amis toujours en opposition, écrivant des lettres anonymes. J'en ai été victime un temps: une amie de très longue date s'est mise à écrire à Roland des lettres anonymes. La jalousie est un sentiment beaucoup plus fréquent qu'on ne le croit, elle peut s'introduire dans n'importe quelle situation, chez n'importe qui. Même chez Nicette, qui est très belle. C'est une chose parfois très difficile à supporter pour un enfant, que l'impression de n'être aimé qu'en surface. Pas pour ses qualités mais pour son apparence. Elle le sent très bien, cette enfant, quand on fait des compliments à une autre.

voIX ET IMAGES Dans le cas de Nicette, c'est plus que de la jalousie. Lorsqu'elle a ravi à Cora l'homme qui s'intéressait à elle, elle ne sait pas quoi en faire. Elle ne l'aime pas vraiment. Elle a surtout cherché à désapproprier son amie. Elle n'était pas jalouse dans le sens que l'amour de l'homme lui était nécessaire.

CLAIRE MARTIN Non, c'était de l'envie. Elle était jalouse de son bien. Elle croyait que Cora et cet homme s'aimaient. Quand elle découvre que Cora n'est probablement pas du tout intéressée à Maurice, qu'elle a quelqu'un d'autre dans sa vie, elle devient furieuse contre Cora. Elle a été flouée de cette espèce de vengeance. Elle est en état de vengeance perpétuelle. C'est une très méchante femme.

VoiX ET IMAGES C'est curieux que la réaction de la critique qui, tout en saluant la constance d'un style qu'elle juge remarquable, n'a pas paru disposée à entrer dans la proposition de l'œuvre; en tout cas pas dans ses zones d'ombre. Elle était comme hypnotisée par un dénouement heureux. Elle n'a pas semblé voir le destin tragique de Nicette à qui est refusée toute satisfaction. Un personnage fuligineux qui vit d'intrigues et qui semble mourir d'une mystérieuse incapacité de parvenir à phagocyter l'être qui s'est tourné vers elle avec une lumineuse affection.

CLAIRE MARTIN Ça m'a beaucoup étonnée. Quand j'ai lu par exemple la chute d'un des articles de nos journaux qui écrit:«si on comparait ce livre à un meuble, ce serait un joli bonheur du jour», je me suis dit que le critique ne s'est pas donné la peine de réfléchir là-dessus. Parfois, on est un peu victime de ce qu'on a déjà fait. Parmi mes nouvelles, il y en a qui sont peut être un petit peu dures, mais la plupart sont plutôt légères, plutôt humoristiques. On les lit et on s'amuse.

voix ET IMAGES Avant de vous quitter, j'aimerais que vous nous disiez un mot du roman en préparation ${ }^{2}$ ?

CLAIRE MARTIN Je ne veux pas vous révéler la profession de mon personnage principal. Il s'agit d'un homme qui, de par son éducation, a été non seulement tenu d'une façon sévère pour l'ordinaire de la vie, mais aussi d'un homme dont on a dès l'enfance décidé l'avenir. Il y a des gens qui ne peuvent pas faire autre chose que ce qu'on les a destinés à faire. Il arrive dans l'endroit où il va faire son métier, si je puis dire, dans un milieu très conventionnel, où il n'est peut-être pas surveillé, mais enfin où on s'occupe de ce qu'il fait. Comme dans tous ces endroits-là, les gens parlent, sont potiniers. Tout le monde s'attend à ce qu'il choisisse une jeune fille et qu'il se marie. Lui attend l'Amour. Il ne veut pas se marier juste pour la forme, il veut vraiment aimer. Mais dans le milieu où il est, le grand amour, vous savez? Puis, un bon jour, il va le rencontrer le grand Amour. Et même la grande passion. Je ne vous dis pas la suite.

2 Il s'appelait Thomas, paru en 2003

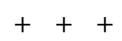




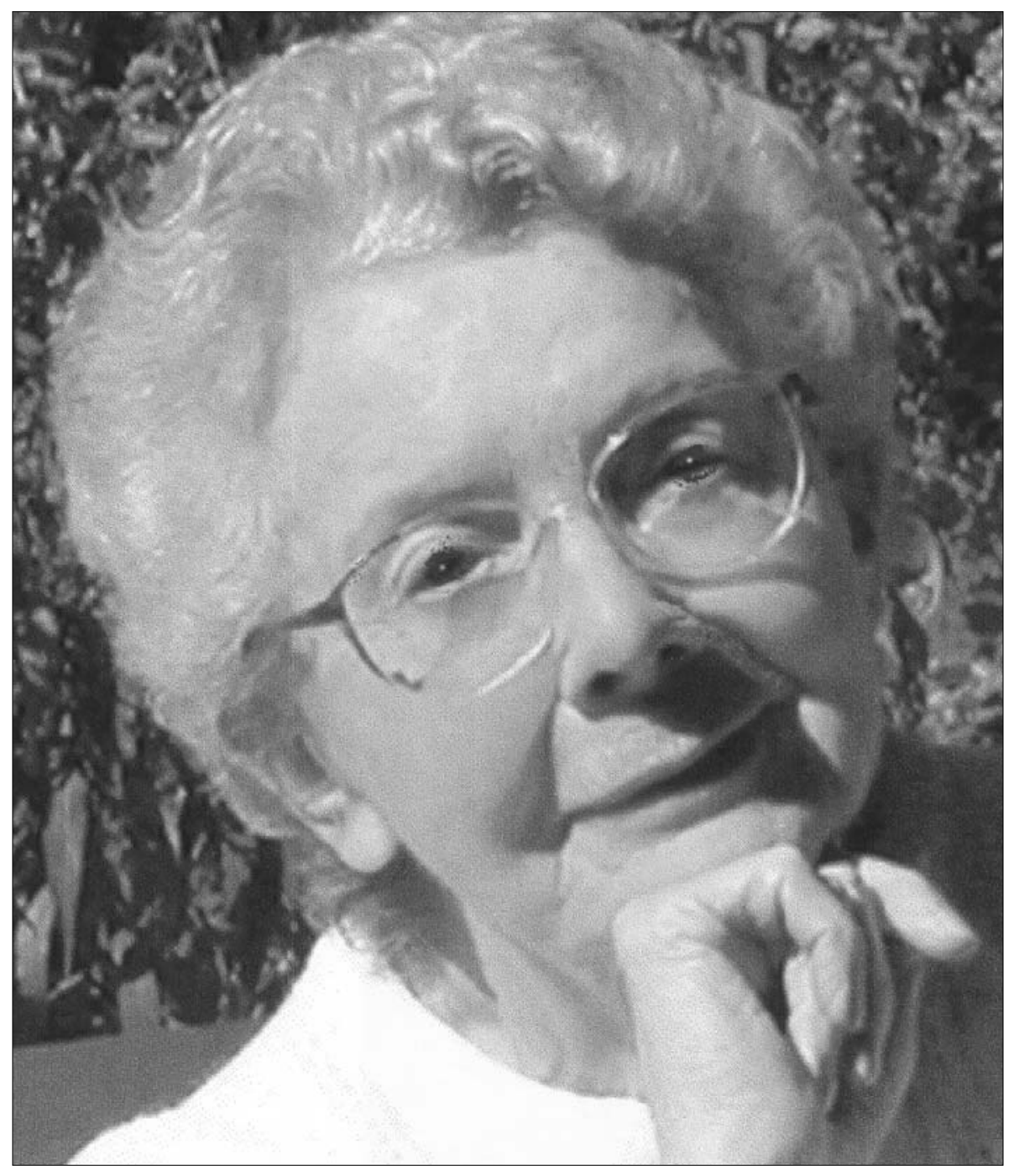

